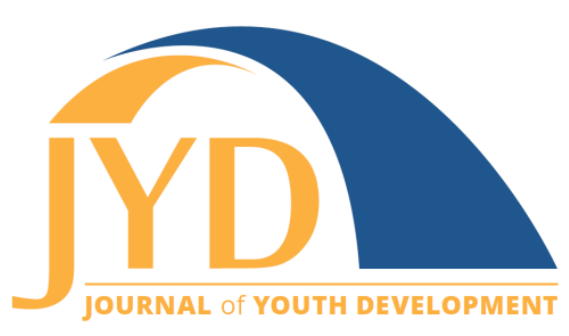

http://jyd.pitt.edu/ | Vol. 15 Issue 3 DOI 10.5195/jyd.2020.944 | ISSN 2325-4017 (online)

\title{
The Role of the Board on Children, Youth, and Families in the Youth Development Field
}

\author{
Angela Diaz \\ Icahn School of Medicine at Mount Sinai and Mount Sinai Adolescent Health Center \\ angela.diaz@mountsinai.org
}

\section{David Britt}

Sesame Workshop, retired

david@britt-cushman.com

James M. Perrin

Harvard Medical School and MassGeneral Hospital for Children

jperrin@mgh.harvard.edu

\section{Abstract}

This special issue of the Journal of Youth Development highlights a range of important contributions that the work of the Board on Children, Youth, and Families (BCYF) at the National Academies of Sciences, Engineering, and Medicine, makes to the field of youth development. This issue traces how BCYF peerreviewed, published consensus reports use transdisciplinary expertise to assemble relevant research, develop evidence-based findings that then undergird policy recommendations, and then communicate them to a wide audience of policy makers, academics, and practitioners. These consensus reports inform and support practice which improves constructive youth development.

Key words: Board on Children, Youth, and Families; public policy; peer review; consensus study; evidence-based; youth development; child development; National Academies of Sciences, Engineering, and Medicine

\section{Introduction}

Articles for this special issue of the Journal of Youth Development were written by staff of the Board on Children, Youth, and Families (BCYF) (in alphabetical order): Emily Backes, Rebekah

(c) $\mathrm{EY}$ New articles in this journal are licensed under a Creative Commons Attribution 4.0 License. This journal is published by the University Library System, University of Pittsburgh and is cosponsored by the University of Pittsburgh Press. The Journal of Youth Development is the official peer-reviewed publication of the National Association of Extension 4-H Agents and the National AfterSchool Association. 
Hutton, Nicole Kahn, Erin Kellogg, Suzanne Le Menestrel, Priyanka Nalamada, and Dara Shefska. Commentaries were authored by BCYF board members David Britt, Richard Catalano, and James Perrin, and immediate past chair of the board Angela Diaz. It was made possible by a Henry and Bryna David Fellowship, which is an internal National Academies of Science, Engineering, and Medicine (the National Academies) program to support staff on a project of their interest.

The articles align BCYF published consensus reports with the field of youth development. BCYF staff is uniquely qualified to undertake these analyses. With overall direction by the BCYF director, staff members lead and provide operational management of each consensus study, from helping define the statement of task, to determining relevant research, drafting and editing the report, and then managing the peer review and communication process.

\section{About BCYF}

BCYF is a joint unit of the Division of Behavioral and Social Sciences and Education (DBASSE) and the Health and Medicine Division of the National Academies. BCYF arose out of earlier work within the National Academies, including the Forum on the Future of Children and Families, the Committee on Child Development and Public Policy, and the Committee on Youth Development.

BCYF board members volunteer their time, energy, expertise, and commitment to the wellbeing of children, youth and families. They bring diverse disciplines and backgrounds, including medicine, prevention, public health, mental health, education, law, and communications, and expertise in research, evaluation, clinical care, and policy.

Among its main work, BCYF undertakes evidence-based, multidisciplinary studies involving medicine, public health, behavioral and social sciences, and education, in order to transform knowledge and analytic tools from these domains into specific findings and recommendations for key public policies, programs, systems, and social services for children, youth, and families. Congress, government agencies, and foundations typically request and sponsor consensus studies and other activities from BCYF and other units of the National Academies.

BCYF engages in work that is both responsive and anticipatory-responsive to government and other sponsors' concerns and questions, and anticipatory of evidence-based approaches that can address major opportunities and challenges in the lives of children, youth, and families. For 
consensus studies, experts in relevant fields are appointed to a committee to work together, pro bono, along with the BCYF staff, to create a report based on scientific evidence and agreement on key findings and recommendations.

BCYF reports have major impact; they spur new research and build or expand new and ongoing fields of study. They have led to new or changed policies, and provided key information, suggestions and new directions for practitioners and researchers. In short, BCYF consensus reports illustrate the power of science to drive research, policy, and practice change.

\section{BCYF Strategic Agenda}

BCYF scans the state of issues, opportunities, and social determinants, as well as policies and practices that impact children, youth, and families and their development over the life course. BCYF's current strategic agenda recognizes that children, youth, and families live in complex environments with dynamic interactions among multiple agents and subsystems, including family units; educational institutions; social services; public safety and justice systems; social, and built environments; ${ }^{1}$ employment; media; public health; and healthcare. BCYF has six strategic priority areas:

1. Healthy development and resilience across physical, cognitive, behavioral, emotional, and social domains for all children and youth-at home, in school, at work, and in communities.

2. Healthy systems, such as social, physical, and built environments that support children, youth, and families.

3. Healthy lifestyles and prevention of physical, emotional, social, and cognitive health issues or toxic stress through family, healthcare, school, and community programs.

4. Comprehensive and integrated care, including acute and chronic care for physical, behavioral, emotional, social, and cognitive health, and for life situations such as pregnancy and perinatal needs.

5. Effective service systems, such as foster care, justice, child welfare, health, education, and employment.

\footnotetext{
${ }^{1}$ According to the Centers for Disease Control and Prevention, the built environment "includes all of the physical parts of where we live and work (e.g., homes, buildings, streets, open spaces, and infrastructure)." See https://www.cdc.gov/nceh/publications/factsheets/impactofthebuiltenvironmentonhealth.pdf
} 
6. Equity, advancing fair and just systems and care for all children, youth, and families to provide opportunities to achieve their goals, and resilience to deal with life course challenges.

The current strategic agenda considers assets, promises and opportunities for children and youths' healthy development and resilience. This is consistent with the approach of the field of positive youth development. BCYF addresses issues through the lens of wellness, healthy lifestyles, prevention, and overall well-being. BYCF understands that positive and negative experiences influence children and families from preconception to young adulthood and are key components of health and well-being over the life course.

The BCYF strategic agenda also emphasizes understanding the role of systems such as the built environment and types of food available in specific neighborhoods in the choices children, youth, and families have. The systems themselves and the environments in which families live need to make the easy choice the healthy choice. Families need the information, resources, and support to avail themselves of healthy choices.

Children, adolescents, and family members interact with multiple systems, such as the health, education, employment, and at times, child welfare, foster care, and juvenile or criminal justice systems. Each of these systems needs to be concerned with positive development and meeting the needs of the population, as well as keeping children and youth safe. All these sectors need to collaborate to help children, youth and families thrive. Health and education are linked. Families need easy access to excellent and integrated early childhood and health services. Children and youth who are not healthy have poorer academic outcomes. Young people who do not have a positive experience with school are less healthy and less prepared to pursue higher education or better employment opportunities.

\section{BCYF's Focus on Equity}

One example of BCYF's focus on equity was a 2015 meeting that discussed determinants of health inequities and social injustice. Participants included researchers, policymakers, community service providers, and representatives who work with vulnerable populations. They addressed

- laws, policies, and leadership to promote social justice and health equity; 
- "institutions" such as parenting, the juvenile justice system, the foster care system, and educational systems, and the ways these institutions could protect the development of children and youth in the context of social justice and health equity;

- health disparities resulting from discriminatory practices and policies, and missed opportunities for investing in human capital; and

- topics and priority areas on equity for the National Academies.

This meeting resulted in the later publication of Perspectives on Health Equity and Social Determinants of Health (Bogard et al., 2017).

\section{BCYF Reports}

Since its creation in 1993, BCYF has published more than 50 consensus reports and has collaborated with other units of the National Academies on more than 60 additional reports. BCYF has hosted gatherings with thought leaders, and fostered discussions across the sectors involved in the well-being of children, youth, and families. The BCYF's publications have informed decisions of policymakers, provided tools for educators and activists, and created new frameworks for understanding the multidisciplinary nature of children's and youths' well-being.

Among BCYF's early and most impactful reports is From Neurons to Neighborhoods: The Science of Early Childhood Development (National Research Council and Institute of Medicine, 2000) chaired by former BCYF Board Chair Jack Shonkoff. ${ }^{2}$ Another recent report by a BCYF consensus committee that promises to be very influential is The Promise of Adolescence: Realizing Opportunity for All Youth, (National Academies of Sciences, Engineering, and Medicine, 2019a) chaired by Richard Bonnie. ${ }^{3}$ Multiple foundations funded this consensus report, which is one of four reports prepared for the National Academy of Medicine's Culture of Health Program supported by the Robert Wood Johnson Foundation (RWJF). A related consensus report, led by the National Academies' Board on Population Health with collaboration with BCYF and funded through the Culture of Health program, addresses early childhood in similar ways and provides recommendations for programs, policies, and systems guided by the

\footnotetext{
2 Jack Shonkoff is Julius B. Richmond FAMRI Professor of Child Health and Development at the Harvard T.H. Chan School of Public Health and the Harvard Graduate School of Education; professor of pediatrics at Harvard Medical School and Boston Children's Hospital; and director of the university-wide Center on the Developing Child at Harvard University.

${ }^{3}$ Richard Bonnie is Harrison Foundation Professor of Medicine and Law and director of the Institute of Law, Psychiatry and Public Policy at the University of Virginia.
} 
newer evidence in the science of early childhood: Vibrant and Healthy Kids: Aligning Science, Practice, and Policy to Advance Health Equity (National Academies of Sciences, Engineering, and Medicine, 2019b). The Culture of Health Program is a multiyear, collaborative effort supported by RWJF to identify strategies to create and sustain conditions that promote equitable good health for all Americans.

This special issue authored by BCYF staff highlights a number of important recent consensus reports. These include

- Preventing Bullying Through Science, Policy, and Practice (National Academies of Sciences, Engineering, and Medicine, 2016)

- Promoting the Educational Success of Children and Youth Learning English: Promising Futures (National Academies of Sciences, Engineering, and Medicine, 2017)

- The Promise of Adolescence: Realizing Opportunity for All Youth (National Academies of Sciences, Engineering, and Medicine, 2019a)

- Shaping Summertime Experiences: Opportunities to Promote Healthy Development and Well-Being for Children and Youth (National Academies of Sciences, Engineering, and Medicine, 2019c)

- Fostering Healthy Mental, Emotional, and Behavioral Development in Children and Youth: A National Agenda (National Academies of Sciences, Engineering, and Medicine, 2019d)

- Promoting Positive Adolescent Health Behaviors and Outcomes: Thriving in the 21st Century (National Academies of Sciences, Engineering, and Medicine, 2020)

For over 25 years, BCYF provided evidence-based recommendations to help children, youth, and families be healthy, educated, resilient, productive, and have a sense of overall well-being. With this special issue, BCYF staff are disseminating findings and recommendations from some of these consensus reports to wider audiences, especially including the positive youth development field.

\section{References}

Bogard, K., Murry, V., \& Alexander, C. (Eds.). (2017). Perspectives on health equity and social determinants of health. National Academy of Medicine.

National Academies of Sciences, Engineering, and Medicine. (2016). Preventing bullying through science, policy, and practice. The National Academies Press. https://doi.org/10.17226/23482 
Journal of Youth Development | http://jyd.pitt.edu/ | Vol. 15 Issue 3 DOI 10.5195/jyd.2020.944

The Role of BCYF in the Youth Development Field

National Academies of Sciences, Engineering, and Medicine. (2017). Promoting the educational success of children and youth learning English: Promising futures. The National Academies Press. https://doi.org/10.17226/24677

National Academies of Sciences, Engineering, and Medicine. (2019a). The promise of adolescence: Realizing opportunity for all youth. The National Academies Press. https://doi.org/10.17226/25388

National Academies of Sciences, Engineering, and Medicine. (2019b). Vibrant and healthy kids: Aligning science, practice, and policy to advance health equity. The National Academies Press. https://doi.org/10.17226/25466

National Academies of Sciences, Engineering, and Medicine. (2019c). Shaping summertime experiences: opportunities to promote healthy development and well-being for children and youth. The National Academies Press. https://doi.org/10.17226/25546

National Academies of Sciences, Engineering, and Medicine. (2019d). Fostering healthy mental, emotional, and behavioral development in children and youth: A national agenda. The National Academies Press. https://doi.org/10.17226/25201

National Academies of Sciences, Engineering, and Medicine. (2020). Promoting positive adolescent health behaviors and outcomes: Thriving in the $21^{\text {st }}$ century. The National Academies Press. https://doi.org/10.17226/25552

National Research Council and Institute of Medicine. (2000). From neurons to neighborhoods: The science of early childhood development. Committee on Integrating the Science of Early Childhood Development. BCYF, Commission on Behavioral and Social Sciences and Education. National Academies Press. 